\title{
HLA expression in a primary uveal melanoma, its cell line, and four of its metastases
}

D J R Blom, L R H M Schurmans, I De Waard-Siebinga, D De Wolff-Rouendaal, J E E Keunen, $M$ J Jager

\begin{abstract}
Background-The level of HLA expression on a tumour may influence the immunological response against this tumour, and vice versa. HLA expression was determined in a primary uveal melanoma, its metastases, and on a cell line derived from this melanoma, and the presence and type of infiltrate in tissue sections were also studied. Methods-Immunohistochemistry with monoclonal antibodies (MAbs) against HLA class I and II, T cells, NK cells, and macrophages.

Results-Primary and metastatic lesions, as well as the cell line showed high levels of expression of the monomorphic determinants of HLA class I. Expression of the polymorphic HLA-A2 and HLA-A3 antigens was decreased on metastases to the skin and liver. HLA-Bw4 expression was low on all lesions, as well as expression of HLA class II. Tumour infiltrating cells consisted mainly of CD3, CD4, and CD8 positive cells. Expression on the cell line corresponded to expression on the primary tumour.

Conclusion-The primary uveal melanoma as well as the cell line showed a high expression of monomorphic and polymorphic HLA-A antigens, while metastases showed a high expression of monomorphic and a lower expression of polymorphic antigens. This variation in expression may support tumour cell escape from NK cells as well as CTL mediated lysis.

(Br f Ophthalmol 1997;81:989-993)
\end{abstract}

Ophthalmology

Leiden University

Hospital, Netherlands

D J R Blom

L R H M Schurmans

I De Waard-Siebinga

J E E Keunen

M J Jager

Department of Ophthalmic Pathology,

Leiden University

Hospital, Netherlands

D De Wolff-Rouendaal

Correspondence to:

D J R Blom, MD,

Department of

Ophthalmology, PO Box

9600, 2300 RC Leiden,

Netherlands.

Accepted for publication 25 June 1997

Changes in HLA expression often occur during carcinogenesis. ${ }^{1}$ Since the function of cytotoxic T lymphocytes (CTLs), as well as of natural killer $(\mathrm{NK})$ cells, is directly influenced by expression of HLA class I antigens, ${ }^{23}$ changes in HLA class I expression might influence an antitumour immune response, and, therefore, prognosis. An association between HLA class I expression and prognosis was observed, for instance, in larynx and breast carcinomas: a lack of HLA class I expression correlated with a more aggressive tumour behaviour and a worse prognosis. ${ }^{4}$ In skin melanoma, the transformation of normal skin melanocytes to melanoma cells is frequently accompanied by a decreased expression of HLA class I antigens, ${ }^{5}$ and complete or selective loss of expression of HLA class I has been observed more frequently in metastatic compared with primary skin melanomas. ${ }^{6}$ Van Duinen et al showed an association between lack of HLA class I expression in locoregional metastases and poor prognosis in patients with stage II cutaneous melanoma. ${ }^{7}$

Although several authors have studied the expression of monomorphic, ${ }^{8-10}$ and polymorphic HLA class I and/or II expression in primary uveal melanomas, ${ }^{11}$ data on HLA expression on metastases of uveal melanoma are still scarce. To our knowledge, only one such study has been performed, in which HLA class I expression was studied on a culture derived from a primary uveal melanoma and on two separate cell cultures derived from liver metastases of the same patient. ${ }^{12}$ In Tran et al's report, it was concluded that both primary and metastatic tumour cells expressed HLA class I.

In the present study, we determined the level of expression of HLA class I and II molecules on a primary intraocular melanoma, on four of its metastases, and on a cell line obtained from the primary tumour. The presence of tumour infiltrating cells in these lesions was also assessed, and compared with the expression of the HLA antigens.

\section{Materials and methods}

CLINICAL AND HISTOPATHOLOGICAL FINDINGS The patient was a 76-year-old woman referred to the department of ophthalmology of Leiden University Hospital with a large tumour in the right orbit extending from the eye (largest tumour diameter $20 \mathrm{~mm}$ ). On examination, the visual acuity of the right eye was 0 and the best corrected visual acuity of the left eye was 0.3 . The right eyeball had been displaced superotemporally by a large tumour, and vessel growth was observed throughout the eyeball.
With the exception of a cortical cataract, the left eye showed no abnormalities. Computed and deformed eyeball with tumour outgrowth into orbital structures. Size and location of the malignancy excluded conservative treatment, and orbital exenteration was performed. Before orbital exenteration, the patient did not receive any treatment.

Following exenteration, the tumour was dissected. One part of the tumour was prepared for tissue culture, and has given rise to the establishment of a well characterised cell line. ${ }^{13}$ Two different parts of the tumour from randomly chosen areas were snap frozen and stored at $-80^{\circ} \mathrm{C}$ until sectioning for immunotomography of the right orbit revealed a small 
Table 1 Quantitative counts of the percentage of cells in sections and cytospots of primary uveal melanoma, and in sections of metastasised uveal melanoma reacting with monoclonal antibodies directed against HLA class I antigens

\begin{tabular}{|c|c|c|c|c|c|c|c|c|}
\hline \multirow[b]{2}{*}{ Antigen } & \multirow[b]{2}{*}{$M A b$} & \multirow{2}{*}{$\frac{\text { Cell line }}{92-1}$} & \multicolumn{2}{|c|}{ Primary tumour } & \multicolumn{4}{|l|}{ Metastases } \\
\hline & & & Part 1 & Part 2 & Adrenal gland & Skin & Liver & Heart \\
\hline$\overline{M e l ~ A s s ~} \mathrm{Ag}^{\star}$ & NKI-beteb & $5 /+$ & $5 /+$ & $5 /++$ & $5 /+$ & $5 /++$ & $5 /+++$ & $5 /+++$ \\
\hline HLA-A,B,C & W6/32 & $5 /++$ & $5 /++$ & $5 /++$ & $5 /+$ & $5 /++$ & $5 /+$ & $5 /++$ \\
\hline $\mathrm{b} 2-\mathrm{mic}$ & BBM1 & $5 /+++$ & $5 /++$ & $5 /++$ & $5 /+$ & $5 /++$ & $5 /+$ & $5 /++$ \\
\hline A locus & HCA2 & $5 /+++$ & $3 /+$ & $5 /+$ & $5 /+$ & $4 /+$ & $3 /+$ & $5 /+$ \\
\hline A2 & MA2.1 & $5 /++$ & $5 /++$ & $5 /++$ & $5 /++$ & $3 /+$ & $1 /++$ & $5 /++$ \\
\hline A3 & GAP-A3 & $5 /++$ & $5 /++$ & $5 /++$ & $5 /+$ & $3 /+$ & $2 /+$ & $4 /+$ \\
\hline B locus & $\mathrm{HC} 10$ & $5 /++$ & $3 /+$ & $5 /++$ & $3 /+$ & $4 /+$ & $4 /+$ & $5 /++$ \\
\hline Bw4 & 116528 & $3 /+$ & $1 /++$ & $1 /+++$ & $2 /++$ & $1 /++$ & $2 /++$ & $1 /++$ \\
\hline $\mathrm{DP}$ & $\mathrm{B} 7 / 21.2$ & 0 & $2 /++$ & $2 /+++$ & $2 /++$ & $2 /++$ & $2 /++$ & $2 /+++$ \\
\hline DQ & SPV-L3 & 0 & $2 /++$ & $2 /++$ & $2 /++$ & $2 /+++$ & $3 /+++$ & $2 /+++$ \\
\hline $\mathrm{DR}$ & B811.2 & 0 & $2 /++$ & $2 /++$ & $2 /++$ & $2 /++$ & $1 /+$ & $2 /++$ \\
\hline
\end{tabular}

${ }^{\star}$ Melanoma associated antigen, as determined with MAb NKI-beteb.

Score: $1=$ less than $5 \%$ of cells staining positive; $2=5-25 \% ; 3=26-50 \% ; 4=51-75 \% ; 5=76-100 \%$.

Intensity of staining: $+=$ slight,$++=$ moderate, and $+++=$ strong staining.

histochemistry. The remainder of the tumour was processed for histopathological examination by an ocular pathologist (D De W-R) (paraffin embedded tissue sections stained with haematoxylin and eosin). Histopathology and immunohistochemistry with the melanoma antigen specific MAb NKI-beteb (Monosan, Uden, Netherlands) revealed a malignant melanoma, with extensive extrabulbar growth. The tumour was histologically classified as an epitheloid uveal melanoma. Mitoses were counted in 15 high power fields $(\mathrm{HPF})$ with a total magnification of $\times 320$, using an eyepiece grid $\left(15 \mathrm{HPF}=4.3 \mathrm{~mm}^{2}\right)$. Two and a half year after exenteration, the patient died as a result of uveal melanoma metastases. Obduction showed multiple melanoma metastases in various sites. Metastatic lesions were dissected from the liver, adrenal gland, the skin, and the heart, and snap frozen and stored at $-80^{\circ} \mathrm{C}$.

HLA TYPING

DNA analysis on peripheral blood leucocytes revealed that the patient had the following HLA type: HLA class I: $A^{\star} 02, A^{\star} 03, A^{\star} 0206$, $\mathrm{B}^{\star} 12, \mathrm{~B}^{\star} 44, \mathrm{~B}^{\star} 05, \mathrm{~B}^{\star} 04, \mathrm{C}^{\star} 0501, \mathrm{C}^{\star} 14$; HLA class II: DR4, DR53, DQ7, DQ8, DQ3.

IMMUNOHISTOCHEMISTRY

For immunohistochemical staining, anti-HLA monoclonal antibodies were used in agreement with the HLA type of the patient (Table 1). References of the anti-HLA MAbs have been described previously. ${ }^{14}$ For infiltrate analysis the following MAbs were used: Leu-4, specific for CD3 (Becton Dickinson, San Jose, CA, USA); RIV6, specific for CD4 (Becton Dickinson); FK18, specific for CD8 (Dako, Glostrup, Denmark); Leu 19, specific for CD $56^{15}$; Dako macrophage, specific for CD68 (Dako). A three step immunoperoxidase technique was employed as described previously. ${ }^{14}$ Negative controls were performed with PBS/bovine serum albumin $1 \%$ (PBS/BSA $1 \%$ ) replacing the primary antibody.

\section{IMMUNOCYTOCHEMISTRY}

HLA expression was determined by immunocytochemistry on cell line 92-1, established from the primary tumour of this patient. ${ }^{13}$ Cells were harvested by trypsinisation $(0.01 \%$ trypsin), and attached to glass slides using a Shandon cytocentrifuge. These cytospin preparations (cytospots) were air dried and fixed for 10 minutes in acetone at room temperature and stored at $-80^{\circ} \mathrm{C}$ until required for cytochemistry. The same procedure for staining with MAbs was followed as for the tissue sections. ${ }^{14}$

ANALYSIS OF ANTIGEN EXPRESSION

For each antibody, the numbers of positively stained tumour cells were estimated and expressed as the percentage of the total number of tumour cells in the analysed section. Percentages were put into five categories $(1=$ $<5 \%, 2=5-25 \%, 3=26-50 \%, 4=51-75 \%, 5$ $=76-100 \%)$. The slides were examined independently by two observers. Interobserver disagreements did not exceed one class, and in case of interobserver disagreement, consensus could be reached during joint evaluation. In addition, the staining pattern of the blood vessels in the tumour was determined for each anti-HLA antibody and classified as positive, uncertain, or negative. Staining for NKI-beteb was used to assess the location of tumour cells in each section. Immunohistochemical results were evaluated without access to histological data.

\section{Results}

Standard histopathology showed that the metastases had the same cell type (that is, epitheloid) as the primary uveal melanoma. The tumour tissue of the metastases showed less coherence than the primary tumour, and little variation in vessel pattern. There were fewer normal and more atypical mitoses in metastases in comparison with the primary tumour (0-5/15 HPF, and 35/15 HPF, respectively).

Immunohistochemical staining was performed with several anti-HLA class I and II MAbs on a primary uveal melanoma and four metastases. In primary as well as metastatic lesions, vessels stained positive with all antiHLA MAbs tested (Table 1). In all tissue sections studied-that is, of the primary tumour as well as of the metastatic lesions, more than $75 \%$ of the tumour cells stained positively with the MAb directed against the melanoma associated antigen NKI-beteb, the anti-HLA class I MAb W6/32, and the anti- $\beta 2$ 


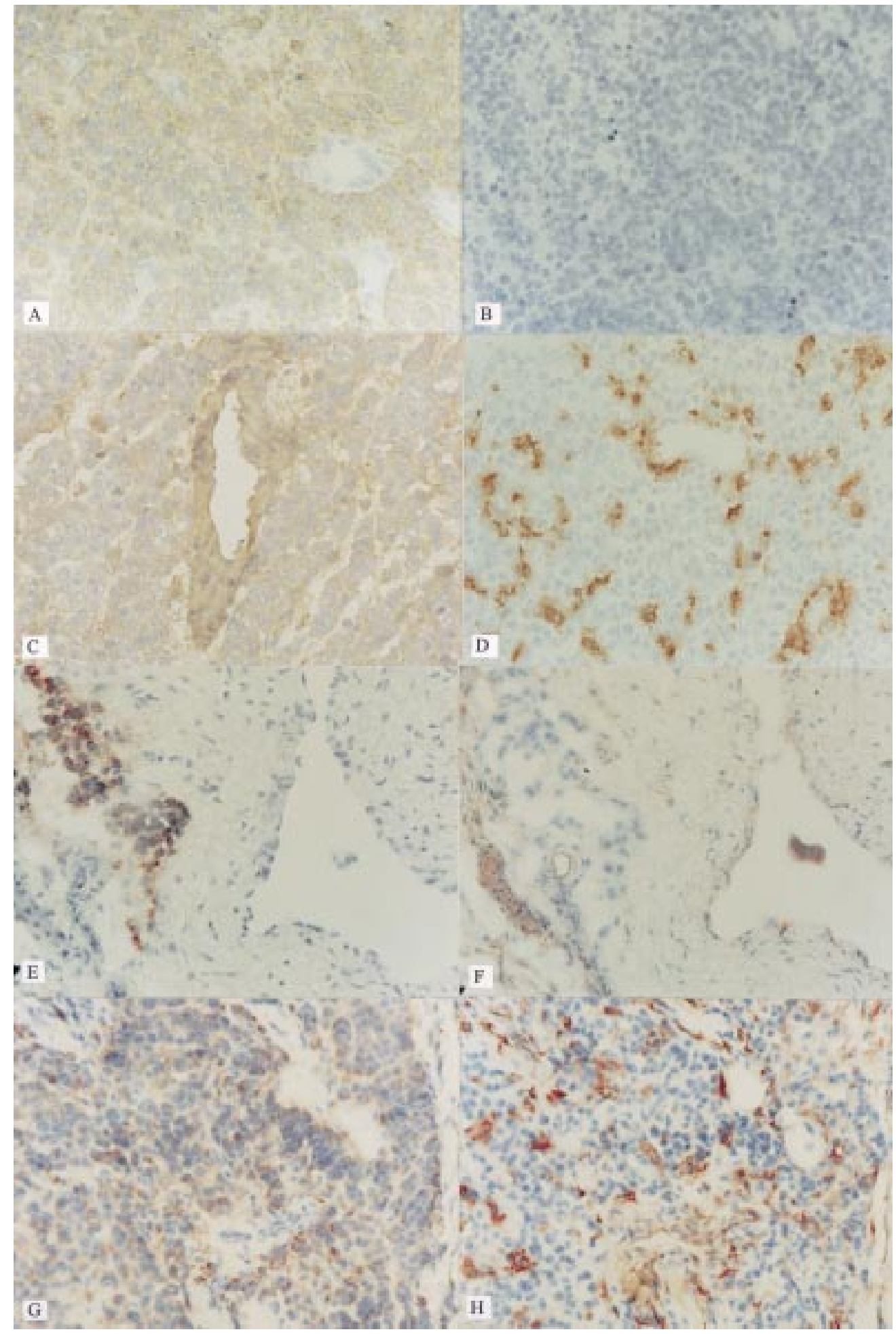

Figure 1 Expression of: (A) NKI-beteb on part one of the primary tumour. All tumour cells stain positively, while vessels are not stained; (B) negative control (PBS) on part one of the primary tumour. No staining is observed; (C) HLA-A2 on part one of the primary tumour. Tumour cells, as well as vessels stain positive; (D) HLA-DR on part one of the primary tumour. Scattered infiltrating cells and part of the tumour cells stain positively; (E) NKI-beteb on the liver metastasis. A cluster of positive staining cells can be discerned near a negative vessel; (F) HLA-A2 on the liver metastasis. Consecutive section to (E) the same cluster of NKI-beteb positive tumour cells does not stain positive for HLA-A2; $(G)$ NKI-beteb on the skin metastasis. Virtually the whole section stains positive, excluding the vessels; (H) HLA-DR on the skin metastasis. Consecutive section to $(G)$ scattered infiltrating cells and part of the tumour cells stain positively. (Original magnification $\times 200$.)

microglobulin MAb BBM1. The MAbs recognising polymorphic antigens showed a more variable pattern. The two different parts obtained from the primary tumour stained similarly, with exception of the anti-A and B locus specific MAbs. One of the parts showed a slightly lower expression with these MAbs. Some of the metastases stained only partly 
Table 2 Amount of tumour infiltrating cells in the lesions, depicted as cells per $\mathrm{mm}^{2}$

\begin{tabular}{|c|c|c|c|c|c|c|}
\hline \multirow[b]{2}{*}{ Cells } & \multicolumn{2}{|c|}{ Primary tumour } & \multicolumn{4}{|l|}{ Metastases } \\
\hline & Part 1 & Part 2 & Adrenal gland & Skin & Liver & Heart \\
\hline $\mathrm{CD}^{+}{ }^{+}$ & 1.2 & 16.4 & 2.8 & 27.7 & 8.8 & 6.5 \\
\hline $\mathrm{CD}^{+}{ }^{+}$ & 2.7 & 1.4 & 1.7 & 20.3 & 5.6 & 1.6 \\
\hline $\mathrm{CD}^{+}$ & 0.6 & 0.4 & 1.1 & 0.9 & 2 & 4.3 \\
\hline $\mathrm{CD} 56^{+}$ & 0 & 0 & 0 & 0 & 0.3 & 0 \\
\hline $\mathrm{CD} 68^{+}$ & 0 & 0 & 0 & 10.7 & 0.4 & 0 \\
\hline
\end{tabular}

positive with the three anti-HLA-A MAbs. Metastases in skin and liver showed a lower expression of HLA-A2 and HLA-A 3 than the primary melanoma and the other two metastases (Fig 1C, 1E, and 1F).

With regard to HLA-B, variable percentages and intensities of staining were found on primary tumour parts as well as on the metastases. The percentage of tumour cells staining positively for HLA-Bw4 did not exceed $25 \%$ in the primary and the metastatic lesions.

For HLA class II, a similarity in staining was observed between primary tumour parts and metastases (Fig 1D, 1G, and 1H), with more than $5 \%$ of the tumour cells staining positively. NKI-beteb positive areas were selected for evaluation. In addition, scattered HLA class II positive non-tumour cells were observed in all sections.

The results of infiltrate analysis with immunohistochemistry are shown in Table 2 . In the primary tumour, the $\mathrm{CD}^{+}$and $\mathrm{CD}^{+}$cells accounted for the majority of tumour infiltrating cells. High densities of $\mathrm{CD}^{+}$and $\mathrm{CD} 4^{+}$ cells were especially found in skin and liver metastases. $\mathrm{CD}^{+}$cells were also observed, although in smaller numbers. No tumour infiltrating $\mathrm{CD} 68^{+}$macrophages or $\mathrm{CD}^{+} 6^{+} \mathrm{NK}$ cells were observed in the two parts of the primary tumour. In the metastatic lesions, a few CD $56^{+}$NK cells were observed in small numbers in the liver metastasis. Some $\mathrm{CD}^{+} 8^{+}$macrophages were observed in the liver and skin lesions. However, CD68 does not recognise all macrophages, but we used this marker to make a comparison of different sites. In all metastatic lesions, $\mathrm{T}$ cells were more frequently observed than NK cells or macrophages.

\section{IMMUNOCYTOCHEMISTRY}

We determined HLA expression on cytospin preparations of cell line 92-1, established from the primary tumour of this patient, ${ }^{13}$ using the same anti-HLA MAbs as for the tissue sections. Expression levels for all anti-HLA class I antigens except $\mathrm{Bw} 4$ were similar to those on the primary tumour parts; the percentage of cells staining for $\mathrm{Bw} 4$ was higher on the cytospot, although with a lower intensity of staining (Table 1). No expression of HLA class II antigens was detected on the cell line.

\section{Discussion}

We compared the expression of HLA class I and II antigens on cells of a primary uveal melanoma, on four of its metastases, and on a cell line obtained from the primary tumour. A high expression of HLA class I was observed on all lesions as well as on the cell line, while expression of HLA class II was low or absent.
De Waard-Siebinga et al ${ }^{111416}$ had previously shown, that polymorphic HLA antigens are often not properly expressed on primary uveal melanomas, but no information was available on the expression of these HLA antigens on uveal melanoma metastases. A previous study by Tran et al showed a high monomorphic HLA class I expression on a short term cell culture derived from a primary uveal melanoma, as well as on two separate cell cultures derived from liver metastases of the same patient. ${ }^{12}$ While we observed high levels of expression of the monomorphic HLA antigens and of $\beta 2$ microglobulin on all lesions, a lower expression of polymorphic HLA-A antigens was observed on one part of the primary tumour and on two of the metastases (to skin and liver), and all lesions showed a very low expression of the polymorphic HLA-Bw4 antigen.

Proper expression of HLA antigens is considered essential for a functional CTL response: lack of expression of HLA antigens is often regarded as a tumour 'escape' mechanism. ${ }^{5}$ For instance, in cutaneous melanoma, a higher frequency of complete loss of expression of HLA class I has been observed in metastatic lesions in comparison with primary tumours. ${ }^{6}$ In a recent study involving a retrospective analysis of 30 patients with uveal melanoma, we observed that high expression of especially HLA-A (and to a lesser extent of HLA-B) on the primary uveal melanoma was strongly correlated with poor patient survival. ${ }^{17}$ This fits with our present finding of a high expression of monomorphic HLA on the primary as well as the metastatic lesions. It may be that the different observations with regard to the relation between HLA expression and survival in cutaneous and uveal melanomas, are associated with differences in the route of metastatis. Uveal melanoma spreads haematogenously, in contrast with cutaneous melanoma, which spreads mainly lymphatically. In these two ways of metastasising, different effector cells may play a role. It is known, for instance, that NK cells are especially equipped to eliminate individual tumour cells in the circulation and thus serve as the earliest cellular effector mechanism against dissemination of blood borne metastases, ${ }^{18}$ while in the skin, CTL are the most effective. Therefore, in uveal melanoma, NK cells may play a role, and cells with a high expression of HLA have a better chance of escaping NK cell lysis. An important role of NK cells in the prevention of uveal melanoma metastases was demonstrated in a mouse model, where disruption of NK cell function significantly increased the development of hepatic metastases from uveal melanoma cells. ${ }^{19}$ Those tumour cells that escape the function of NK cells, might give rise to metastases which will be positive for class I.

In our study, the expression of primary tumour and metastases did not differ for class II expression. Interestingly, although more than $5 \%$ of the cells of primary as well as metastatic lesions stained positive for class II determinants, only a small number of inflammatory tumour infiltrating cells was observed, which 
could not account for the HLA class II staining. In previous studies, other authors also reported only small numbers of infiltrating cells in uveal melanoma. ${ }^{16}{ }^{20}$ Remarkably, cell line 92-1 did not demonstrate any HLA class II expression. The observation that a proportion of melanoma cells are HLA-DR positive is not surprising, since in our laboratory Jager et al ${ }^{9}$ and De Waard-Siebinga et $a l^{14}$ have previously demonstrated that sections of human uveal melanoma express HLA class II antigens.

A possible explanation for the expression of HLA class II on human uveal melanoma sections, is the influence of (cytokines produced by) tumour infiltrating cells on antigen expression. This is further supported by the finding of De Waard-Siebinga, who observed that uveal melanoma cells grown in vitro lost expression of the class II determinant HLADR, while expression of HLA class I on cultured melanoma cells remained similar to the expression on the original tumour. ${ }^{14}$ In conclusion, our present data are in line with previous studies ${ }^{12} 17$ that support the following theory: because of their mainly haematogenous route of metastasis, uveal melanoma cells with a high expression of monomorphic HLA class I have a better chance to evade NK cell mediated lysis and, hence, are well suited to give rise to metastases. In the tissues, CTLs may attack the melanoma cells, as long as they show a good expression of HLA class I. Immunotherapy may specifically be aimed against these metastases.

The authors thank Professor Dr Th M Vroom and R Broekhuizen from the Department of Pathology, Academic Hospital Utrecht, Netherlands, for kindly supplying the monoclonal antibodies HCA2 and HC10. We are grateful to S Van De Burg of the Department of Immunohematology and Bloodbank, Leiden University Hospital, for kindly supplying the monoclonal antibodies W6/32 and BBM1, and Dr Gelsthorpe, Sheffield, UK, for kindly supplying the monoclonal antibodies 116.5.28, and SFR8B6. This study was supported in part by the Haagsch Oogheelkundig Fonds and the Stichting Blinden-Penning.

1 Garrido F, Cabrera T, Concha A, Glew S, Ruiz-Cabello F, Stern PL. Natural history of HLA expression during tumour development. Immunol Today 1993;14:491-9.

2 Kaufman DS, Schoon RA, Robertson MJ, Leibson PJ. Inhibition of selective signaling events in natural killer cells rec- ognizing major histocompatibility complex class I. Proc Natl Acad Sci 1995;92:6484-8.

3 Gumperz JE, Parham P. The enigma of the natural killer cell. Nature 1995;378:245-8.

4 Concha A, Esteban F, Cabrera T, Ruiz-Cabello F, Garrido $\mathrm{F}$. Tumor aggresiveness and MHC class I and II antigens in laryngeal and breast cancer. Cancer Biol 1991;2:47-54.

5 Ferrone S, Marincola FM. Loss of HLA class I antigens by melanoma cells: molecular mechanisms, functional significance and clinical relevance. Immunol Today 1995;16:48794.

6 Ruiter DJ, Mattijssen V, Bröcker EB, Ferrone S. MHC antigens in human melanomas. Semin Cancer Biol 1991;2:3545

7 Van Duinen SG, Ruiter DJ, Bröcker EB, Van Der Velde EA, Sorg C, Welvaart K, et al. Level of HLA antigens in locoregional metastases and clinical course of the disease in patients with melanoma. Cancer Res 1988;48:1019-25.

8 Jager MJ, De Wolff-Rouendaal D, Breebaart AC, Ruiter DJ. Expression of HLA antigens in paraffin sections of uveal melanomas. Doc Ophthalmol 1986;64:69-76.

9 Jager MJ, Van Der Pol JP, De Wolff-Rouendaal D, De Jong PTVM, Ruiter DJ. Decreased expression of HLA class II antigens on human uveal melanoma cells after in vivo X-ray irradiation. Am f Ophthalmol 1988;105:78-86.

10 Natali PG, Bigotti A, Nicotra MR, Nardi RM, Delovu A, Segatto $\mathrm{O}$, et al. Analysis of the antigenic profile of uveal melanoma lesions with anti-cutaneous melanomaassociated antigen and anti-HLA monoclonal antobodies. Cancer Res 1989;49:1269-74.

11 De Waard-Siebinga I, Houbiers JGA, Hilders CGJM, De Wolff-Rouendaal D. Differential expression of HLA-A and -B alleles on uveal melanoma as determined by immunohistology. Ocul Immunol Inflamm 1996;4:1-14.

12 Tran JM, Murray TG, Uno T, Valore N, Ksander BR. Expression of HLA class I on ocular melanoma during progression from primary to metastatic disease. ARVO Abstracts. Invest Ophthalmol Vis Sci 1996;37:S208.

13 De Waard-Siebinga I, Blom DJR, Griffioen M, Schrier PI, Hoogendoorn E, Beverstock G, et al. Establishment and characterization of an uveal melanoma cell line. Int f Cancer 1995;62:155-61

14 De Waard-Siebinga I, Kool J, Jager MJ. HLA antigen expression on uveal melanoma cells in vivo and in vitro. Hum Immunol 1995;44:111-7.

15 Lanier LL, Testi R, Bindl L, Phillips JH. Identity of Leu-19 (CD56) leucocyte differentiation antigen and neural cell adhesion molecule. F Exp Med 1989;169:2233-8.

16 De Waard-Siebinga I, Hilders CGJM, Hansen BE, Delft JL van, Jager MJ. HLA expression and tumor-infiltrating immune cells in uveal melanoma. Graefes Arch Clin Exp Ophthalmol 1995;234:34-42.

17 Blom DJR, Luyten GPM, Mooy CM, Kerkvliet S, Zwinderman AH, Jager MJ. HLA class I expression: a marker of poor prognosis in uveal melanoma. Invest Ophthalmol Vis poor prognosis in
Sci 1997 ; in press.

18 Whiteside TL, Herberman RB. The role of natural killer cells in immune surveillance of cancer. Curr Opin Immunol 1995;7:704-10

19 Ma D, Luyten GP, Luider TM, Niederkorn JY. Relationship between natural killer cell susceptibility and metastasis of human uveal melanoma cells in a murine model. Invest Ophthalmol Vis Sci 1995;36:435-41.

20 Whelchel JC, Farah SE, McLean IW, Burnier MN. Immunohistochemistry of infiltrating lymphocytes in uveal malignant melanoma. Invest Ophthalmol Vis Sci 1993;34: 2603-6. 